Voix et Images

volxetimages

\title{
Les enjeux de la Rencontre internationale Jack Kerouac
}

\section{Rémi Ferland}

Volume 13, numéro 3 (39), printemps 1988

Jack Kerouac et l’imaginaire québécois

URI : https://id.erudit.org/iderudit/200731ar

DOI : https://doi.org/10.7202/200731ar

Aller au sommaire du numéro

\section{Éditeur(s)}

Université du Québec à Montréal

\section{ISSN}

0318-9201 (imprimé)

1705-933X (numérique)

Découvrir la revue

\section{Citer cet article}

Ferland, R. (1988). Les enjeux de la Rencontre internationale Jack Kerouac. Voix et Images, 13(3), 422-425. https://doi.org/10.7202/200731ar d'utilisation que vous pouvez consulter en ligne.

https://apropos.erudit.org/fr/usagers/politique-dutilisation/ 


\title{
Les enjeux de la Rencontre internationale Jack Kerouac
}

\author{
par Rémi Ferland, Université Laval
}

Un simple coup d'œil sur la volumineuse revue de presse que vient de publier le Secrétariat permanent des peuples francophones, organisateur de la récente Rencontre internationale Jack Kerouac, nous convaincra de ne pas nous limiter à un compte rendu, sous peine de tomber dans la redite et le lieu commun. Aussi tâcherai-je, non tant de résumer la manifestation que, succinctement, de montrer la genèse de ces quatre journées et d'estimer le fruit qu'en ont pu tirer les études kérouaciennes d'ici.

Il faut d'abord rappeler que le Club Jack Kerouac a été fondé en avril 1984, avec, comme objectif principal, l'étude de l'individualité canadienne-française de Kerouac. Louis Dussault, directeur du Secrétariat permanent des peuples francophones, organisme tutélaire, a d'ailleurs expliqué en détail, dans le troisième bulletin N'importe quelle route, les raisons d'être et la pertinence d'un tel regroupement 1 . Car il pouvait sembler paradoxal à première vue qu'un organisme voué à la promotion de la langue et de la culture françaises en Amérique s'associe au nom d'un écrivain qui, pour francophone qu'il fût en famille, n'en a pas moins écrit toute son œuvre en anglais. Mais précisément, ce Kerouac ambivalent pouvait servir de trait d'union entre le Québec et la Franco-Américanie aussi bien qu'entre le Québec et l'Amérique anglophone, et par là contribuer à une diffusion et à une meilleure connaissance de notre identité.

C'est dans cet esprit que Louis Dupont et Eric Waddell, tous deux géographes et spécialistes de l'Amérique française, établirent les bases de la Rencontre internationale Jack Kerouac. Je m'associai d'emblée à ce projet, car comme lecteur de Kerouac, j'avais toujours trouvé un surcroît de plaisir dans les rapprochements évidents entre son expérience et la nôtre. Cependant, la valeur de Kerouac écrivain reste indéniable et il ne fallait pas réduire son œuvre à un document historique ou sociologique, si riche fût-elle sous cet aspect. C'est ainsi qu'ont été définis les quatre grands thèmes de la Rencontre (identité, éthique, écriture, influence), avec le souci d'une variété totalisante qui permettrait à chacun, selon son champ d'investigation, d'y trouver son profit. L'œuvre d'art n'est-elle pas, selon le mot d'Umberto Eco, une auvre ouverte? Aucune lecture ne devrait donc être exclusive.

1 Louis Dussault, «Le Secrétariat permanent des peuples francophones et la rencontre internationale Jack Kerouac», $N^{\prime}$ importe quelle route, vol. $1, \mathrm{n}^{\circ} 3$, p. 8-9. 
De fait, quand on passe en revue la liste des participants à la Rencontre, on s'aperçoit que la diversité était au rendez-vous, car contrairement aux quelques autres manifestations de ce genre par le passé, on a pu entendre non seulement des amis beats de Kerouac, mais aussi bien des écrivains influencés par son œuvre, des professeurs et des chercheurs de tous horizons.

Cet éclatement théorique s'est révélé dans les conférences certes, mais davantage lors des tables rondes, où des points de vue souvent contradictoires se cristallisaient de manière radicale et irréversible. La première journée a donné lieu aux échanges les plus soutenus et les moins conciliants: chacun tenait à faire sien Kerouac, à l'associer en exclusivité à telle collectivité, au détriment d'une autre. Franco-Américains, Québécois, Américains, Franco-Ontariens aussi bien, le voyaient à leur façon comme un des leurs. Par delà la partisanerie parfois aveugle, ces appropriations révélaient la richesse de l'expérience kérouacienne, véritable carrefour d'orientations culturelles. C'est ce que soulignait en particulier la conférence d'Eric Waddell, opinément placée en conclusion de la Rencontre et dans laquelle, en un regard théorique distancié, neutre et globalisant, étaient évoqués les différents substrats collectifs à l'œuvre chez Kerouac.

D'autres interventions sont à retenir, pour ce qu'elles ont apporté de neuf et d'important. À l'ouverture du colloque littéraire, Roger Brunelle, FrancoAméricain de Lowell a retracé en détail l'enfance de Kerouac, un aspect plutôt négligé ou méconnu jusqu'alors et pourtant essentiel. Il l'a fait avec l'exactitude méticuleuse d'un archiviste doublé d'un détective. La tâche n'était pas aisée. Le Kerouac de cette époque déjà lointaine se confond dans une masse anonyme fort peu répertorice par l'Histoire. Mais Roger Brunelle a su mettre au jour des témoignages immédiats et des documents surprenants, comme cette photo du petit Jean-Louis Kerouac costumé pour une saynète d'école paroissiale. Qui plus est, autour de ces croquis pris sur le vif, s'est déployé en volutes tout le contexte d'une enfance à Lowell, évocation émouvante pour les participants québécois, qui retrouvaient là et leur mémoire première et sa transposition sur les bords de la rivière Merrimack, comme le songe d'une vie antérieure toute proche mais inexprimée. Par ailleurs, le lendemain, Denis Vanier, écrivain limite de la contreculture, osait dire de façon explicite, dans des expressions d'un lyrisme visionnaire, la conspiration dont a longtemps souffert Kerouac. Tout en dressant un parallèle audacieux avec ce qui se passe au niveau poétique ici au Québec, sa conférence a donné lieu à force métaphores et antonomases démonstratives: soldat Lebrun des grandes orgues, cowboy bouddhiste, poète red-neck, génial heavymetal des années 50. Ces formules vraies et troublantes résument en quelques mots, à la façon d'haiku, la vie et le drame de Kerouac et nul à ma connaissance n'aurait pu les produire avec autant de bonheur, sinon Kerouac lui-même. Auparavant le même jour, Josée Yvon, romancière au féminisme plutôt controversé, critique littéraire à l'ironie allègre et corrosive, n'avait pas manqué non plus d'originalité et de hardiesse, en s'attaquant aux «tendances sexuelles de Jack Kerouac». Car si ouverte que se prétende notre époque, certains sujets, curieusement, restent délicats à aborder, même dans un tel colloque. Les normes du conscient collectif ne se laissent pas transgresser aisément. Ainsi, malgré une 
souplesse relative de l'organisation, nous avons pu craindre parfois, comme participants, de collaborer malgré nous à une entreprise de récupération: canalisés par un pouvoir occulte, nous allions, à notre insu, produire pour des fins officielles un Kerouac inoffensif, conforme à ce que doit être tout écrivain accrédité. Si le complot a jamais pu exister (il faut être vigilant), la conférence de Josée Yvon a contribué, comme d'autres, à l'enrayer et nous avons eu droit à une lecture franche et sans tutelle. Il n'est pas jusqu'aux témoignages d'Allen Ginsberg et de Carolyn Cassady, pourtant cent fois sollicités par des enquêtes du genre, qui n'aient apporté aussi quelque chose d'inédit. Si les faits mentionnés n'étaient pas toujours inconnus, il étaient dits du moins de façon renouvelée. Tout s'est passé en effet comme si, placés dans un contexte inhabituel, ces deux amis de longue date de Kerouac le considéraient tout à coup sans a priori, avec le même étonnement interrogatif que nous tous.

En regard et même parfois à l'intérieur de ces études approfondies sur Kerouac, une tendance nouvelle s'est affirmée, qui restera, je crois, comme une des contributions remarquables de cette Rencontre d'octobre 1987. Sans minimiser ce qui, çà et là, a pu déjà se faire dans le même sens, mais isolément, il me semble en effet que l'on a vraiment accordé pour la première fois une importance spécifique à l'écrivain Kerouac, sans lier nécessairement sa pratique d'écriture à sa vie. Sa biographie fascine et séduit, à bon droit, pour toutes les raisons déjà évoquées. Mais durant la Rencontre, on est passé aussi à un autre plan de lecture, comme le souhaitait Kerouac lui-même. Il en est résulté d'autres interventions tout aussi inattendues, par exemple celles de Maurice Poteet, sur la métatextualité, de Jacques Houbart, sur la traduction d'un cryptolangage, ou de Gilles Farcet, sur l'ascendance littéraire de Kerouac.

Je ne viens de rappeler que quelques noms et pour être juste, il faudrait relire tous les Actes, un plaisir auquel nous convieront sous peu les éditions Guérin. Comme je n'ai pu assister à toutes les conférences, mes omissions n'ont donc rien de significatif (que dire des interventions de Jean-Maurice Morisset ou de Pier Vittorio Tondelli dont les intitulés figuraient déjà tout un programme: «Jack Kerouac et Louis Riel (le Canada errant à la recherche de lui-même)» et «Le travail des jeunes écrivains italiens en rapport à la culture du rock et de la Beat Generation»).

Comme lecteur de Kerouac, on ne peut que se réjouir du succès de cette Rencontre. Pourtant, il faut aussi, quitte à gâcher ce plaisir, s'interroger sur les causes de ce soudain engouement pour Kerouac. L'écrivain satisfait-il un certain défaitisme post-référendaire? S'agirait-il, implicitement, de persuader une nation qu'elle peut fort bien vivre sa culture sans la langue qui lui est propre? Pour outrées qu'elles puissent paraître, ces questions sont d'actualité. Elles viennent spontanément, par exemple quand on lit telle déclaration du secrétaire de l'association Action pour les Franco-Américains du Nord-Est (ACFANE), selon qui la personnalité culturelle franco-américaine en Nouvelle-Angleterre paraît 
suffisamment forte pour se perpétuer sans la connaissance du français ${ }^{2}$. Voilà qui est troublant et peut être grave de conséquences. Les Franco-Américains et les Québécois ne pensent pas tous ainsi et, comme Roger Brunelle, il faut souhaiter pour les siens et bientôt pour nous-mêmes un Kerouac francophone 3 .

Mais, en définitive, ce qui se dégage de ces quatre jours qui feront date désormais dans la recherche kérouacienne, c'est que l'œuvre résiste fort bien à tous les cribles auxquels on l'a soumise. Encore intacte et inviolée, resplendissante par delà les commentaires, elle s'offre toujours libre au lecteur. On a poussé certaines incursions parfois fort avant, mais il suffit d'ouvrir certains titres de Kerouac, comme Book of Dreams ou Visions of Gerard pour voir des terrae incognita défiler devant nous, fertiles, se dérobant encore à la critique. Voilà de quoi nous rassurer si besoin était: d'autres études viendront encore nous parler de cet écrivain majeur de sa génération.

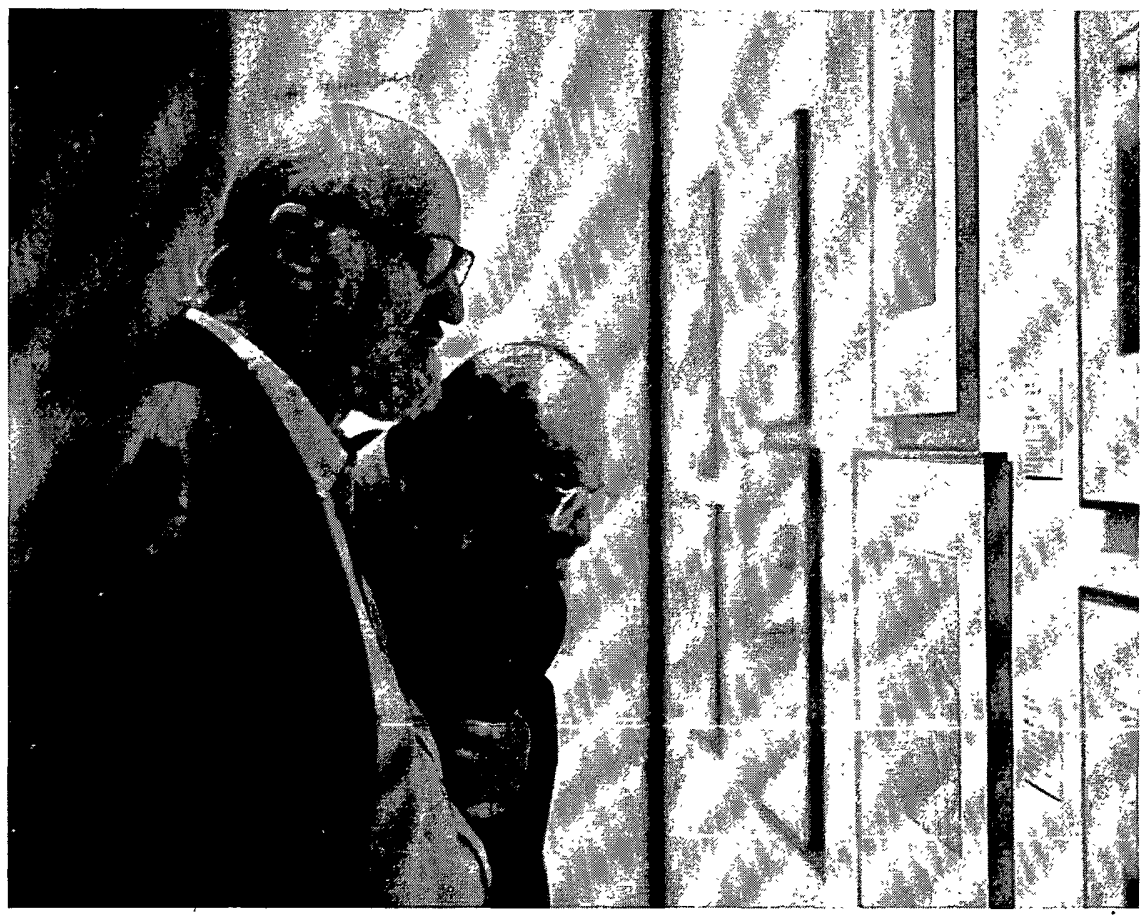

Lawrence Ferlinghetti et Allen Ginsberg scrutant les photos de Robert Frank à l'exposition «Canuck et clochard céleste: l'univers de Jack Kerouac», octobre 1987 (Photo: Musée du Québec)

2 Paul Paré cité par J.P. Péroncel-Hugoz, «Des Américains doublement immigrés: les "Francos" de Nouvelle-Angleterre», le Monde, 2 septembre 1987, p. 3.

3 Ibid. 